PROCEEDINGS OF THE

AMERICAN MATHEMATICAL SOCIETY

Volume 125, Number 1, January 1997, Pages 153-158

S 0002-9939(97)03640-X

\title{
CONVEX SOLUTIONS OF THE SCHRÖDER EQUATION IN BANACH SPACES
}

\author{
JANUSZ WALORSKI
}

(Communicated by J. Marshall Ash)

\begin{abstract}
The problem of the existence and uniqueness of increasing and convex solutions of the Schröder equation, defined on cones in Banach spaces, is examined on a base of the Krein-Rutman theorem.
\end{abstract}

The aim of this paper is to obtain a theorem on the existence and uniqueness of increasing and convex solutions $\varphi$ of the Schröder equation

$$
\varphi(f(x))=\rho \varphi(x),
$$

one of the most important equations of linearization, having many applications in various fields of mathematics (see [4] and [5]). Our result generalizes the theorem of F.M. Hoppe [1], in particular for functions defined on infinite-dimensional Banach spaces. The main point is to obtain an infinite-dimensional analogue of $[2$, Theorem 1] by A. Joffe and F. Spitzer exploiting the famous Krein-Rutman theorem [3, pp. 267-270], cf. also [6, Theorem 2.1].

\section{Preliminaries}

Fix a non-degenerate Banach space $(X,\|\cdot\|)$ and a closed cone $K \subset X$ with non-empty interior, i.e. (cf. [3, p. 217, Definition 2.1]), $K$ is a closed subset of $X$ such that $K+K \subset K, t K \subset K$ for every $t \geq 0, K \cap(-K)=\{\theta\}$ and $\operatorname{Int} K \neq \emptyset$. We define a (partial) order $\leq$ on $X$ by $x \leq y$ iff $y-x \in K$, and we assume that the norm $\|\cdot\|$ is an increasing function on $K$, i.e. $\theta \leq x \leq y$ implies $\|x\| \leq\|y\|$. (According to [7, p. 216], if $X$ is a real space and there exists a real constant $\gamma \geq 1$ such that $\theta \leq x \leq y$ implies $\|x\| \leq \gamma\|y\|$, then in the space $X$ there exists an equivalent norm which is increasing on $K$.)

Let $A: X \rightarrow X$ be a completely continuous linear operator such that $A K \subset K$ and for every $x \in K \backslash\{\theta\}$ there exists a positive integer $n$ such that $A^{n} x \in \operatorname{Int} K$. By the Krein-Rutman theorem [3, p. 267] the spectral radius $\rho$ of $A$ is positive and there exists exactly one vector $u \in \operatorname{Int} K$ and exactly one continuous linear functional $g: X \rightarrow \mathbb{R}$ such that $A u=\rho u, g(A x)=\rho g(x)$ for every $x \in X, g(x)>0$ for every $x \in K \backslash\{\theta\},\|u\|=1$ and $g(u)=1$. Moreover [3, p. 269-270], the spectral radius of the operator $B: X \rightarrow X$ defined by

$$
B x=A x-\rho g(x) u
$$

Received by the editors September 12, 1994 and, in revised form, July 1, 1995.

1991 Mathematics Subject Classification. Primary 39B52, 39B12, 39B22.

Key words and phrases. Schröder functional equation, convex and increasing solutions, KreinRutman theorem.

(C)1997 American Mathematical Society 
is less than $\rho$ and

$$
\left\|\rho^{-n} A^{n} x-g(x) u\right\| \leq \rho^{-n}\left\|B^{n}\right\|\|x\| \quad \text { for } n \in \mathbb{N} .
$$

We assume also that a function $f: K \rightarrow K$ is given and such that

$$
\begin{gathered}
f(x) \neq \theta \quad \text { for } x \in K \backslash\{\theta\}, \\
\lim _{x \rightarrow \theta}(f(x)-A x) /\|x\|=\theta
\end{gathered}
$$

and there exists a positive $c$ such that

$$
g(x) \geq c\|g\|\|x\| \quad \text { for } x \in f(K) .
$$

Let us note that in the case where $X$ is finite-dimensional the last condition is always satisfied.

\section{The Joffe-Spitzer Sequence}

The main result of this section reads:

Theorem 1. Assume that either

or

$$
\rho<1
$$

If $x_{0} \in K \backslash\{\theta\}$ and $\lim _{n \rightarrow \infty} f^{n}\left(x_{0}\right)=\theta$, then

$$
\lim _{n \rightarrow \infty} f^{n}\left(x_{0}\right) / g\left(f^{n}\left(x_{0}\right)\right)=u .
$$

Proof. Fix $r_{0}>0$ such that the closed ball centered at $u$ with the radius $r_{0}$ is contained in $K$. Then

$$
x \leq r_{0}^{-1}\|x\| u \quad \text { for } x \in X .
$$

Put

$$
\alpha_{n}:=\rho^{-n}\left\|B^{n}\right\|\left(c r_{0}\|g\|\right)^{-1} \quad \text { for } n \in \mathbb{N} .
$$

According to the last part of the Krein-Rutman theorem

$$
\lim _{n \rightarrow \infty} \alpha_{n}=0
$$

and

$$
\rho^{n}\left(1-\alpha_{n}\right) g(x) u \leq A^{n} x \leq \rho^{n}\left(1+\alpha_{n}\right) g(x) u
$$

for every positive integer $n$ and $x \in f(K)$. Define $F: K \rightarrow X$ by

$$
F(x):=f(x)-A x
$$

and put

$$
\beta_{n}:=\left(c r_{0}\|g\|\right)^{-1}\left\|F\left(f^{n}\left(x_{0}\right)\right)\right\| /\left\|f^{n}\left(x_{0}\right)\right\| \quad \text { for } n \in \mathbb{N} .
$$

It follows from (2) and (3) that

$$
\lim _{n \rightarrow \infty} \beta_{n}=0
$$

We shall show

$$
\pm A^{n-k-1} F\left(f^{k+m}\left(x_{0}\right)\right) \leq \rho^{n-k-1} \beta_{k+m} g\left(f^{k+m}\left(x_{0}\right)\right) u \leq \rho^{n-k-1} \beta_{k+m} g\left(f^{m}\left(x_{0}\right)\right) u
$$


for $n \in \mathbb{N}, k \in\{0, \ldots, n-1\}$ and $m$ large enough, say $m>M$.

Applying (7) and (4) we obtain

$$
\pm F\left(f^{k+m}\left(x_{0}\right)\right) \leq \beta_{k+m} g\left(f^{k+m}\left(x_{0}\right)\right) u \quad \text { for } k, m \in \mathbb{N} .
$$

Hence, as $A$ increases,

$$
\begin{aligned}
\pm A^{n-k-1} F\left(f^{k+m}\left(x_{0}\right)\right) & \leq \beta_{k+m} g\left(f^{k+m}\left(x_{0}\right)\right) A^{n-k-1} u \\
& =\rho^{n-k-1} \beta_{k+m} g\left(f^{k+m}\left(x_{0}\right)\right) u
\end{aligned}
$$

for $m, n \in \mathbb{N}$ and $k \in\{0, \ldots, n-1\}$. To get the right-hand-side of (13) assume first (5), fix a $\lambda \in(\rho, 1)$ and, making use of $(3), \delta>0$ such that

$$
\|F(x)\| /\|x\| \leq(\lambda-\rho) c r_{0}\|g\| \quad \text { for } x \in K \text { with } 0<\|x\| \leq \delta .
$$

Then, applying also (7),

$$
F(x) \leq(\lambda-\rho) g(x) u \quad \text { for } x \in f(K) \text { with } 0<\|x\| \leq \delta,
$$

whence

$$
g(f(x))=g(A x)+g(F(x)) \leq \lambda g(x) \quad \text { for } x \in f(K) \text { with } 0<\|x\| \leq \delta .
$$

Now, if $M$ is a positive integer such that $\left\|f^{m}\left(x_{0}\right)\right\| \leq \delta$ for $m>M$, then

$$
g\left(f^{k+m}\left(x_{0}\right)\right) \leq \lambda^{k} g\left(f^{m}\left(x_{0}\right)\right) \leq g\left(f^{m}\left(x_{0}\right)\right)
$$

for $m>M$. This jointly with (14) ends the proof of (13) in case (5). In case (6) we have $g(f(x)) \leq g(A x)=g(x)$ for $x \in K$ which jointly with (14) gives (13).

Since

$$
f^{n}(x)=A^{n} x+\sum_{k=0}^{n-1} A^{n-k-1} F\left(f^{k}(x)\right) \quad \text { for } n \in \mathbb{N}, x \in K,
$$

it follows from (10) that

$$
\begin{array}{r}
\rho^{n}\left(1-\alpha_{n}\right) g(x) u+\sum_{k=0}^{n-1} A^{n-k-1} F\left(f^{k}(x)\right) \leq f^{n}(x) \\
\leq \rho^{n}\left(1+\alpha_{n}\right) g(x) u+\sum_{k=0}^{n-1} A^{n-k-1} F\left(f^{k}(x)\right)
\end{array}
$$

for $n \in \mathbb{N}, x \in f(K)$. Using these inequalities for $x=f^{m}\left(x_{0}\right)$ and applying (13) we get

$$
\begin{gathered}
{\left[\rho^{n}\left(1-\alpha_{n}\right)-\sum_{k=0}^{n-1} \rho^{n-k-1} \beta_{k+m}\right] g\left(f^{m}\left(x_{0}\right)\right) u \leq f^{m+n}\left(x_{0}\right)} \\
\leq\left[\rho^{n}\left(1+\alpha_{n}\right)+\sum_{k=0}^{n-1} \rho^{n-k-1} \beta_{k+m}\right] g\left(f^{m}\left(x_{0}\right)\right) u
\end{gathered}
$$

for $n \in \mathbb{N}$ and $m>M$. Let $N$ be a positive integer such that $\alpha_{n}<1$ for $n>N$, and for each $n>N$ let $M_{n}>M$ be a positive integer such that

$$
\rho^{n}\left(1-\alpha_{n}\right)-\sum_{k=0}^{n-1} \rho^{n-k-1} \beta_{k+m}>0 \quad \text { for } m>M_{n} .
$$


Making use of (15) and the facts that $g$ increases and $g(u)=1$ we obtain

$$
\begin{aligned}
-2 \frac{\alpha_{n}+\sum_{k=0}^{n-1} \rho^{-(k+1)} \beta_{k+m}}{1-\alpha_{n}-\sum_{k=0}^{n-1} \rho^{-(k+1)} \beta_{k+m}} u & \leq-2 \frac{\alpha_{n}+\sum_{k=0}^{n-1} \rho^{-(k+1)} \beta_{k+m}}{1+\alpha_{n}+\sum_{k=0}^{n-1} \rho^{-(k+1)} \beta_{k+m}} u \\
\leq \frac{f^{m+n}\left(x_{0}\right)}{g\left(f^{m+n}\left(x_{0}\right)\right)}-u & \leq 2 \frac{\alpha_{n}+\sum_{k=0}^{n-1} \rho^{-(k+1)} \beta_{k+m}}{1-\alpha_{n}-\sum_{k=0}^{n-1} \rho^{-(k+1)} \beta_{k+m}} u
\end{aligned}
$$

for $n>N, m>M_{n}$. Moreover,

$$
-y \leq x \leq y \text { implies }\|x\| \leq 3\|y\| \quad \text { for } x, y \in X .
$$

Consequently,

$$
\left\|\frac{f^{m+n}\left(x_{0}\right)}{g\left(f^{m+n}\left(x_{0}\right)\right)}-u\right\| \leq 6 \frac{\alpha_{n}+\sum_{k=0}^{n-1} \rho^{-(k+1)} \beta_{k+m}}{1-\alpha_{n}-\sum_{k=0}^{n-1} \rho^{-(k+1)} \beta_{k+m}}
$$

for $n>N$ and $m>M_{n}$. Hence and from (12) we get

$$
\limsup _{m \rightarrow \infty}\left\|\frac{f^{m}\left(x_{0}\right)}{g\left(f^{m}\left(x_{0}\right)\right)}-u\right\| \leq 6 \frac{\alpha_{n}}{1-\alpha_{n}} \quad \text { for } n>N
$$

which jointly with (9) ends the proof.

Corollary 1. Under the assumptions of Theorem 1 we have

$$
\lim _{n \rightarrow \infty} f^{n}\left(x_{0}\right) /\left\|f^{n}\left(x_{0}\right)\right\|=u
$$

and

$$
\lim _{n \rightarrow \infty}\left\|f^{n+1}\left(x_{0}\right)\right\| /\left\|f^{n}\left(x_{0}\right)\right\|=\rho .
$$

\section{The Szekeres Sequence}

Passing to solutions of (S) we assume additionally that the function $f$ is increasing, convex and

$$
\lim _{n \rightarrow \infty} f^{n}(x)=\theta \quad \text { for } x \in K \backslash\{\theta\} .
$$

Observe that then in such a case zero is the only fixed point of $f$ and

$$
\lim _{n \rightarrow \infty} g\left(f^{n+1}(x)\right) / g\left(f^{n}(x)\right)=\rho \quad \text { for } x \in K \backslash\{\theta\} .
$$

Fix arbitrarily an $a \in \operatorname{Int} K$. We shall show that for every $x \in K$ the sequence $\left(g\left(f^{n}(x)\right) / g\left(f^{n}(a)\right)\right)_{n \in \mathbb{N}}$ is bounded in order to define the function $\varphi_{0}: K \rightarrow[0, \infty)$ by the formula

$$
\varphi_{0}(x):=\limsup _{n \rightarrow \infty} g\left(f^{n}(x)\right) / g\left(f^{n}(a)\right) .
$$

In fact, if $x \in K \backslash\{\theta\}$, then $f^{N}(x) \leq a$ for a positive integer $N$. Consequently, $g\left(f^{n}\left[f^{N}(x)\right]\right) \leq g\left(f^{n}(a)\right)$ and, on the other hand,

$$
\frac{g\left(f^{n+N}(x)\right)}{g\left(f^{n+N}(a)\right)}=\frac{g\left(f^{n}\left[f^{N}(x)\right]\right)}{g\left(f^{n}(a)\right)} \prod_{k=1}^{N} \frac{g\left(f^{n+k-1}(a)\right)}{g\left(f^{n+k}(a)\right)} \quad \text { for } n \in \mathbb{N} .
$$

Hence and from (16) we obtain $\limsup _{n \rightarrow \infty} g\left(f^{n}(x)\right) / g\left(f^{n}(a)\right) \leq \rho^{-N}$.

Arguing as F.M. Hoppe did in [1], but using our Theorem 1 instead of [2, Theorem 1] by A. Joffe and F. Spitzer, we can prove what follows. 
Theorem 2. If $\rho<1$, then $\varphi_{0}$ is an increasing and convex solution of (S) and if $\varphi: K \rightarrow \mathbb{R}$ is an increasing and convex solution of $(\mathrm{S})$, then

$$
\varphi(x)=\varphi(a) \varphi_{0}(x) \quad \text { for } x \in K .
$$

Corollary 2. If $\rho<1$, then

$$
\varphi_{0}(x)=\lim _{n \rightarrow \infty} g\left(f^{n}(x)\right) / g\left(f^{n}(a)\right) \quad \text { for } x \in K .
$$

Applying Theorem 1 and Corollary 2 we obtain also a representation of the solution $\varphi_{0}$ in which the functional $g$ does not occur.

Corollary 3. If $\rho<1$, then

$$
\varphi_{0}(x)=\lim _{n \rightarrow \infty}\left\|f^{n}(x)\right\| /\left\|f^{n}(a)\right\| \quad \text { for } x \in K .
$$

Example. Let $I$ denote the interval $[0,1], X$ denote the Banach space of all continuous real functions on $I$ with the supremum norm and $K$ denote the cone of all non-negative functions on $X$. Let $a: I^{2} \rightarrow(0,1)$ be a continuous function. It is easy to check that the function $f: K \rightarrow K$ given by the formula

$$
f(x)(t):=\int_{0}^{1}\left[a(s, t)+\frac{x(s)}{1+x(s)}\right] x(s) d s
$$

satisfies all the assumptions of our theorems, with

$$
A x(t):=\int_{0}^{1} a(s, t) x(s) d s \quad \text { for } t \in I \text { and } x \in X,
$$

except, maybe, condition (4). To get (4) let us observe that putting $\gamma=\inf a(T \times T)$ we have

$$
f(x)(t) /\|f(x)\| \geq \frac{\gamma}{2}=: c>0 \quad \text { for } t \in I
$$

and for every $x \in K \backslash\{\theta\}$. In other words, the ball centered at $f(x) /\|f(x)\|$ and with the radius $c$ is contained in $K$ for every $x \in K \backslash\{\theta\}$. This jointly with [3, p. 210, Lemma 1.2] proves (4).

Remarks. 1. For the sake of simplicity we considered functions defined on the whole cone $K$ but similar results hold if we replace $K$ by $\{x \in K: x \leq a\}$, or by $\{x \in K: x<a\}$, with $a \in \operatorname{Int} K$.

2. Assuming that the function $f$ is concave we can consider increasing and concave solutions of (S) replacing in the definition of $\varphi_{0}$ the upper limit by the lower limit.

\section{ACKNOWLEDGEMENT}

This research was supported by the State Committee for Scientific Research Grant No. 210629101.

\section{REFERENCES}

1. F.M. Hoppe, Convex solutions of a Schröder equation in several variables, Proc. Amer. Math. Soc. 64 (1977), 326-330. MR 56:1486

2. A. Joffe and F. Spitzer, On Multitype Branching Processes with $\rho \leq 1$, J. Math. Anal. Appl. 19 (1967), 409-430. MR 35:3760

3. M.G. Krein and M.A. Rutman, Linear operators leaving invariant a cone in a Banach space, Uspehi Matematičeskih Nauk, (N.S.) 3 , no. 1 (23) (1948), 3-95. [English translation: Functional Analysis and Measure Theory, American Mathematical Society Translations - Series 1, vol. 10, 1962.] MR 10:256c; MR 12:341b 
4. M. Kuczma, Functional equations in a single variable, Monografie Matematyczne 46, PWN Polish Scientific Publishers 1968. MR 37:4441

5. M. Kuczma, B. Choczewski, and R. Ger, Iterative Functional Equations, Encyclopedia of mathematics and its applications 32, Cambridge University Press 1990. MR 92f:39002

6. R.D. Nussbaum, Hilbert's projective metric and iterated nonlinear maps, Memoirs of the American Mathematical Society 391 (1988). MR 89m:47046

7. H.H. Schaefer, Topological Vector Spaces, Graduate Texts in Mathematics 3, Springer-Verlag 1971. MR 49:7722

Instytut Matematyki, Uniwersytet Śląski, Ul. Bankowa 14, PL-40-007 Katowice, Poland

E-mail address: walorski@gate.math.us.edu.pl 